\title{
AN AMALGAMATION OF THE BANACH SPACES ASSOCIATED WITH JAMES AND SCHREIER, PART II: BANACH-ALGEBRA STRUCTURE
}

\author{
ALISTAIR BIRD \\ Department of Mathematics and Statistics, Lancaster University \\ Lancaster, LA1 4YF, United Kingdom \\ E-mail: a.bird@lancs.ac.uk
}

\begin{abstract}
The James-Schreier spaces, defined by amalgamating James' quasi-reflexive Banach spaces and Schreier space, can be equipped with a Banach-algebra structure. We answer some questions relating to their cohomology and ideal structure, and investigate the relations between them. In particular we show that the James-Schreier algebras are weakly amenable but not amenable, and relate these algebras to their multiplier algebras and biduals.
\end{abstract}

1. Introduction. In this paper we introduce the James-Schreier algebras, $V_{p}$ for $p \geqslant 1$, which are, by design, Banach sequence algebras with a bounded approximate identity $\left(V_{p}\right.$ is introduced as a Banach space in [2]). We follow the approach of Andrew and Green [1] and Dales [5, 4.1.45] in their investigations of the algebra structure of the James space $J_{2}$, and White's thesis [13] (alternatively [14]) to that of $J_{p}$ for $p>1$. These previous studies of the James algebras have appeared in a purely Banach-algebra context-1 aspires to a 'basis-free' approach (though relates some results to basis concepts in remarks). In contrast, we present the underlying Schauder basis theory and shift operators on the Banach space as a virtue. Not only does this approach offer efficiency, but perhaps insight: it enables us to frame the non-amenability of the Banach algebra $V_{p}$ in terms of various shift operators on $V_{p}$.

The James and James-Schreier algebras share many common features, despite the fact that $J_{p}$ is $l_{p}$-saturated, whereas $V_{p}$ is $c_{0}$-saturated (see [2, 5.2]). As $V_{p}$ is not quasireflexive, the role of $J_{p}$ in different situations is now performed by one of the two algebras $V_{p}$ and its non-separable counterpart $W_{p}[2,4.2]$. In particular we show that $V_{p}$, like $J_{p}$, is

2010 Mathematics Subject Classification: Primary 46J20; Secondary 46B45, 46J35.

Key words and phrases: amenability, Banach sequence algebra, bounded approximate identity, ideal, James-Schreier algebra, Schreier algebra, weak amenability.

The paper is in final form and no version of it will be published elsewhere. 
weakly amenable but not amenable; and we show that the bidual of $V_{p}$, and the multiplier algebras of $V_{p}$ and $W_{p}$ are all $*$-isomorphic to $W_{p}$ with an adjoined identity.

We also offer a brief glimpse of the Schreier algebras, both as toy counterparts to the James-Schreier algebras, and because they embed as complemented ideals in the corresponding James-Schreier algebras, thus giving one possible means of demonstrating that $V_{p}$ is not amenable.

We make liberal use of definitions and results from the companion Banach-space paper [2] in which the James-Schreier spaces are introduced, though now only concentrate on the scalar field $\mathbb{K}=\mathbb{C}$ for the Banach-algebra case. In particular, for $m \in \mathbb{N}$ and $\zeta \subseteq \mathbb{N}$, we make use of: the unit vectors $\left(e_{n}\right)_{n \in \mathbb{N}}$ in $c_{00}$; the $m^{\text {th }}$ coordinate functional, $f_{m}: \mathbb{C}^{\mathbb{N}} \rightarrow \mathbb{C}:\left(\alpha_{n}\right)_{n \in \mathbb{N}} \mapsto \alpha_{m}$; the natural projection associated with $\zeta, P_{\zeta}$; the $m^{\text {th }}$ natural projection, $P_{m}: \mathbb{C}^{\mathbb{N}} \rightarrow c_{00}:\left(\alpha_{n}\right)_{n \in \mathbb{N}} \mapsto\left(\alpha_{1}, \ldots, \alpha_{m}, 0,0, \ldots\right)$; the element $\chi_{\zeta}$ of $\mathbb{C}^{\mathbb{N}}$ defined by $\left\langle\chi_{\zeta}, f_{k}\right\rangle=1$ if $k \in \zeta$, and 0 otherwise; and in this paper, we write $\chi_{m}$ for $\chi_{(0, m]}$. We note that $\chi_{\zeta}=P_{\zeta} \chi_{\mathbb{N}}$ and $\chi_{m}=P_{m} \chi_{\mathbb{N}}$. The dual of a Banach space $E$ is denoted by $E^{\prime}$, the second dual by $E^{\prime \prime}$.

2. Banach-algebra theory. By a basis for a Banach space, we always understand a Schauder basis.

Proposition 2.1 ([11, 4.2.20]). Any Banach space with a normalised 1-unconditional basis is a commutative non-unital Banach algebra under the pointwise product.

The lemma and norm that follow, may be found in greater generality in the proofs of [5. 2.1.9] or [12, 1.1.9].

Lemma 2.2. Let $A$ be an algebra with identity $e$ and a complete norm $\|\cdot\|$ such that the product $A \times A \rightarrow A$ is separately continuous. Then $\|a\|:=\sup \{\|a b\|: b \in A,\|b\| \leqslant 1\}$ defines an equivalent norm on $A$ such that $\|e\|=1$ and $\|a b\| \leqslant\|a\|\|b\| \quad(a, b \in A)$.

Equip $\mathbb{C}^{\mathbb{N}}$ with the pointwise defined *-algebra operations. A Banach algebra $B$ is a Banach sequence algebra (on $\mathbb{N}$ ) if $B$ is a subalgebra of $\mathbb{C}^{\mathbb{N}}$, and $B$ contains $c_{00}$. For a Banach sequence algebra $B$, and $\zeta \subseteq \mathbb{N}$, we define $I_{B}(\zeta):=\overline{\operatorname{span}}\left\{e_{n}: n \in \zeta\right\}$; this is a closed ideal in $B$.

Lemma 2.3. The closure of $c_{00}$ in a Banach sequence algebra $B$ on which $*$ is a continuous involution, is a $*$-ideal of $B$.

Proof. It is clear that $c_{00}$ is a $*$-ideal in $\mathbb{C}^{\mathbb{N}}$ and hence also in $B$. The closure of $c_{00}$ in $B$ will hence also be a $*$-ideal of $B$.

The following proposition may be found in [5, 4.1.35(i)], however, we give a short direct proof:

Proposition 2.4. Let $B$ be a Banach sequence algebra in which $c_{00}$ is dense. Then the character space of $B$ is $\left\{\left.f_{i}\right|_{B}: i \in \mathbb{N}\right\}$, that is, $B$ is natural.

Proof. Let $\varphi$ be a character on $B$. Then $\varphi\left(\chi_{\zeta}\right) \in\{0,1\}$ for all finite $\zeta \subset \mathbb{N}$, by multiplicativity of $\varphi$. As $\varphi$ is non-zero, $\varphi\left(e_{m}\right)=1$ for some $m \in \mathbb{N}$, and by linearity, since $\chi_{\{m, n\}}=e_{m}+e_{n}$, we must have $\varphi\left(e_{n}\right)=0$ for all $n \neq m$. Hence $\varphi=\left.f_{m}\right|_{B}$. 
Proposition 2.5. Suppose that B is a Banach sequence algebra with a basis. Then:

(i) Any closed ideal in $B$ is of the form $I_{B}(\zeta)$ for some $\zeta \subseteq \mathbb{N}$, and $\left(\chi_{\zeta \cap(0, n]}\right)_{n \in \mathbb{N}}$ is an approximate identity for $I_{B}(\zeta)$;

(ii) $B$ has a bounded approximate identity if and only if $\left(\chi_{n}\right)_{n \in \mathbb{N}}$ is uniformly normbounded.

Proof. (i) This is a restatement of [5, 4.1.35(ii)] or [13, 1.8.3].

(ii) It is clear that if $\left\|\chi_{k}\right\|$ is uniformly bounded for $k \in \mathbb{N}$, then the approximate identity $\left(\chi_{n}\right)_{n \in \mathbb{N}}$ is a bounded approximate identity.

Conversely, let $\left(\omega_{\lambda}\right)$ be a bounded approximate identity with $C:=\sup _{\lambda}\left\|\omega_{\lambda}\right\|<\infty$, and suppose for a contradiction that $\sup _{k}\left\|\chi_{k}\right\|=\infty$. Every Banach space with a basis has a finite basis constant [11, 4.1.17], so we have $M:=\sup _{n}\left\|P_{n}\right\|<\infty$. By our assumption there exists $k \in \mathbb{N}$ such that $\left\|\chi_{k}\right\| \geqslant M C+1$. As $\left(\omega_{\lambda}\right)$ is an approximate identity, there exists $\lambda$ such that $\left\|\chi_{k}-\omega_{\lambda} \chi_{k}\right\| \leqslant \frac{1}{2}$. So for such a $k$ and $\lambda$ we have

$$
\begin{aligned}
\frac{1}{2} & \geqslant\left\|\chi_{k}-\omega_{\lambda} \chi_{k}\right\| \geqslant\left\|\chi_{k}\right\|-\left\|\omega_{\lambda} \chi_{k}\right\|=\left\|\chi_{k}\right\|-\left\|P_{k} \omega_{\lambda}\right\| \\
& \geqslant\left\|\chi_{k}\right\|-M\left\|\omega_{\lambda}\right\| \geqslant(M C+1)-M C=1
\end{aligned}
$$

which results in the required contradiction.

Definition 2.6. Let $A$ be an algebra, and $E$ an $A$-bimodule. A linear map $\delta: A \rightarrow E$ is a derivation if it satisfies $\delta(a b)=a \cdot \delta(b)+\delta(a) \cdot b \quad(a, b \in A)$. A derivation $\delta: A \rightarrow E$ is inner if there exists an element $x \in E$ such that $\delta(a)=a \cdot x-x \cdot a \quad(a \in A)$.

A Banach algebra $B$ is amenable if every bounded derivation from $B$ into a dual Banach $B$-bimodule is inner. A Banach algebra $B$ is weakly amenable if every bounded derivation from $B$ into the dual module $B^{\prime}$ is inner.

Proposition 2.7 ([5, 2.8.72]). Suppose that B is a commutative Banach algebra such that the span of its idempotents is dense in $B$. Then $B$ is weakly amenable.

In particular, a Banach sequence algebra in which $c_{00}$ is dense, is weakly amenable.

For a Banach algebra $B$ with dual module $B^{\prime}$, the Arens products are defined as follows: for $a \in B, f \in B^{\prime}$, and $F, G \in B^{\prime \prime}$, we set $\langle a, F \cdot f\rangle:=\langle F, f \cdot a\rangle$ and $\langle a, f \cdot F\rangle:=$ $\langle F, a \cdot f\rangle$ where $a \cdot f$ and $f \cdot a$ are the usual dual actions; then $\langle F \square G, f\rangle:=\langle F, G \cdot f\rangle$ and $\langle F \diamond G, f\rangle:=\langle G, f \cdot F\rangle$. It is well known that the bidual equipped with either Arens product, $\left(B^{\prime \prime}, \square\right)$ or $\left(B^{\prime \prime}, \diamond\right)$, is a Banach algebra - see [5, 2.6.15] or [4, 9.13(v)]. We say that $B$ is Arens regular if the Arens products coincide.

For $B$ a Banach *-algebra we may define the linear involution $\triangleleft$ on $B^{\prime}$ by setting $\left\langle x, f^{\triangleleft}\right\rangle:=\overline{\left\langle x^{*}, f\right\rangle}$ for all $x \in B$ and $f \in B^{\prime}$. If $B$ is an Arens regular Banach *-algebra then we equip $B^{\prime \prime}$ with the involution $F \mapsto F^{*}$ defined by: $\left\langle F^{*}, f\right\rangle:=\overline{\left\langle F, f^{\triangleleft}\right\rangle}$ for all $F \in B^{\prime \prime}$ as in [5, 3.1.18].

For a Banach space $E$ with a basis $\left(b_{n}\right)_{n \in \mathbb{N}}$, define $\|x\|_{\text {bip }(E)}:=\sup _{m \in \mathbb{N}}\left\|\sum_{n=1}^{m} \alpha_{n} b_{n}\right\|_{E}$ for $x=\left(\alpha_{n}\right)_{n \in \mathbb{N}} \in \mathbb{C}^{\mathbb{N}}$, and $\operatorname{bip}(E):=\left\{x \in \mathbb{C}^{\mathbb{N}}:\|x\|_{\operatorname{bip}(E)}<\infty\right\}$ as in [2, 2.4]; then $\operatorname{bip}(E)$ is a Banach space with respect to the norm $\|\cdot\|_{\operatorname{bip}(E)}$. In the case where $B$ is a Banach sequence algebra in which $\left(e_{n}\right)_{n \in \mathbb{N}}$ is a basis, bip $(B)$ will be a subalgebra of $\mathbb{C}^{\mathbb{N}}$, 
and when $*$ is an isometric involution on $B, *$ will be an isometric involution on $\operatorname{bip}(B)$ as well.

LEMmA 2.8. Let $B$ be a Banach sequence algebra and suppose that $\left(e_{n}\right)_{n \in \mathbb{N}}$ is a shrinking basis for $B$. The isomorphism $\Upsilon: B^{\prime \prime} \rightarrow \operatorname{bip}(B)$ given by $\Upsilon(F):=\left(\left\langle F, f_{n}\right\rangle\right)_{n \in \mathbb{N}}\left(F \in B^{\prime \prime}\right)$, previously defined in [2, 2.4], is an algebra homomorphism when $B^{\prime \prime}$ is equipped with either Arens product, and $B$ is Arens regular. If, additionally, $B$ is a Banach *-algebra with respect to the canonical involution on $\mathbb{C}^{\mathbb{N}}$, then $\Upsilon$ is a*-homomorphism.

Proof. We have $\Upsilon(F \square G)=\Upsilon(F) \cdot \Upsilon(G)$ as

$$
\left\langle F \square G, f_{n}\right\rangle=\left\langle F, G \cdot f_{n}\right\rangle=\left\langle F,\left\langle G, f_{n}\right\rangle f_{n}\right\rangle=\left\langle F, f_{n}\right\rangle\left\langle G, f_{n}\right\rangle \quad(n \in \mathbb{N})
$$

and similarly $\Upsilon(F \diamond G)=\Upsilon(F) \cdot \Upsilon(G)$.

In the Banach $*$-algebra case, let $x=\sum_{i} \alpha_{i} e_{i} \in B$, and $n \in \mathbb{N}$; then we have $\left\langle x, f_{n}^{\triangleleft}\right\rangle=$ $\overline{\left\langle x^{*}, f_{n}\right\rangle}=\overline{\left\langle\sum_{i} \bar{\alpha}_{i} e_{i}, f_{n}\right\rangle}=\alpha_{n}=\left\langle x, f_{n}\right\rangle$. So $f_{n}^{\triangleleft}=f_{n}$ for all $n \in \mathbb{N}$. Thus we conclude that $\Upsilon\left(F^{*}\right)=\left(\left\langle F^{*}, f_{n}\right\rangle\right)_{n \in \mathbb{N}}=\left(\overline{\left\langle F, f_{n}^{\triangleleft}\right\rangle}\right)_{n \in \mathbb{N}}=\left(\overline{\left\langle F, f_{n}\right\rangle}\right)_{n \in \mathbb{N}}=\Upsilon(F)^{*}$.

3. The Schreier spaces as Banach algebras. For the remainder of this paper, we let $p \in[1, \infty), \zeta \subset \mathbb{N}$, and $x:=\left(\alpha_{n}\right)_{n \in \mathbb{N}} \in \mathbb{C}^{\mathbb{N}}$. We recall the following definitions from [2, Section 3]: a set $\zeta$ is admissible if $\operatorname{card}(\zeta) \leqslant \min \zeta$; the seminorm $\mu_{p}(\cdot, \zeta)$ is given by $\mu_{p}(x, \zeta):=\left(\sum_{n \in \zeta}\left|\alpha_{n}\right|^{p}\right)^{1 / p}$; the norm $\|\cdot\|_{Z_{p}}$ is given by $\|x\|_{Z_{p}}:=\sup \left\{\mu_{p}(x, \zeta)\right.$ : $\zeta$ admissible\}; and the two Banach spaces associated with this norm are the unrestricted Schreier space, $Z_{p}:=\left\{x \in \mathbb{C}^{\mathbb{N}}:\|x\|_{Z_{p}}<\infty\right\}$ and the Schreier space, $S_{p}:=$ $\overline{\operatorname{span}}\left\{e_{n}: n \in \mathbb{N}\right\} \subset Z_{p}$.

As the Schreier space $S_{p}$ has a normalised unconditional basis, it is a Banach algebra by Proposition 2.1, and is weakly amenable by Proposition 2.7. A simple calculation gives $\left\|\chi_{n}\right\|_{Z_{p}} \rightarrow \infty$, hence by Proposition 2.5 (ii) we have the following:

COROLlary 3.1. The Schreier algebra $S_{p}$ has no bounded approximate identity.

The lack of a bounded approximate identity prevents $S_{p}$ from being amenable [5. 2.9.57].

Corollary 3.2. The unrestricted Schreier space $Z_{p}$ is a Banach sequence algebra with isometric involution, and $S_{p}$ is an *-ideal of $Z_{p}$.

Proof. As $S_{p}$ is a Banach algebra with isometric involution $*$ and basis $\left(e_{n}\right)_{n \in \mathbb{N}}, \operatorname{bip}\left(S_{p}\right)$ is a Banach algebra and $*$ is an isometric involution on $\operatorname{bip}\left(S_{p}\right)$. Lemma 2.3 implies that $S_{p}$ is a $*$-ideal in $\operatorname{bip}\left(S_{p}\right)$. The result now follows from the fact that $\operatorname{bip}\left(S_{p}\right)=Z_{p}$, , $3.13]$.

By Lemma 2.8, as the Schreier space $S_{p}$ has a monotone shrinking basis [2, 3.5], its bidual $S_{p}^{\prime \prime}$ is Arens regular and is $*$-isomorphic to the Banach $*$-algebra $Z_{p}$.

4. The James-Schreier spaces as Banach $*$-algebras. We recall the following definitions from [2, Sections 2 and 4]: a set $\zeta$ is permissible if $2 \leqslant \operatorname{card} \zeta \leqslant 1+\min \zeta$; the seminorm $\nu_{p}(\cdot, \zeta)$ is given by $\nu_{p}(x, \zeta):=\left(\sum_{j=1}^{k}\left|\alpha_{n_{j}}-\alpha_{n_{j+1}}\right|^{p}\right)^{1 / p}$ for $\zeta=\left\{n_{1}<\cdots<n_{k+1}\right\}$; the norm $\|\cdot\|_{W_{p}}$ is given by $\|x\|_{W_{p}}:=\sup \left\{\nu_{p}(x, \zeta): \zeta\right.$ permissible $\}$; this is a complete 
norm on both the unrestricted James-Schreier space, $W_{p}:=\left\{x \in c_{0}:\|x\|_{W_{p}}<\infty\right\}$, and the James-Schreier space, $V_{p}:=\overline{\operatorname{span}}\left\{e_{n}: n \in \mathbb{N}\right\} \subset W_{p}$.

We let $X_{p}:=\operatorname{bip}\left(V_{p}\right)$ with norm $\|\cdot\|_{X_{p}}:=\|\cdot\|_{\operatorname{bip}\left(V_{p}\right)}$, and recall that $X_{p}=W_{p}+\mathbb{C} \chi_{\mathbb{N}} \cong$ $V_{p}^{\prime \prime}[2,4.15]$, due to the shrinking basis of $V_{p}$ (proved in [2, 4.12] in the case $p>1$, and [3] for $p=1)$. We write $\|\cdot\|_{V_{p}}$ when we consider $\|\cdot\|_{W_{p}}$ restricted to $V_{p}$.

LEMmA 4.1. The Banach space $X_{p}$ is a sub-*-algebra of $\mathbb{C}^{\mathbb{N}}$ with jointly continuous product and isometric involution.

Proof. Making use of a standard inequality given in [2, equation (4.1)], it follows that

$$
\begin{aligned}
|\alpha \beta-\gamma \delta|^{p}=|\alpha \beta-\alpha \delta+\alpha \delta-\gamma \delta|^{p} & \leqslant 2^{p-1}\left(|\alpha \beta-\alpha \delta|^{p}+|\alpha \delta-\gamma \delta|^{p}\right) \\
& =2^{p-1}\left(|\alpha|^{p}|\beta-\delta|^{p}+|\alpha-\gamma|^{p}|\delta|^{p}\right)
\end{aligned}
$$

for any $\alpha, \beta, \gamma, \delta \in \mathbb{C}$.

Thus for $x, y \in W_{p}$ we have $\nu_{p}(x y, \zeta)^{p} \leqslant 2^{p-1}\left(\|x\|_{\infty}^{p} \nu_{p}(y, \zeta)^{p}+\|y\|_{\infty}^{p} \nu_{p}(x, \zeta)^{p}\right)$. Taking the supremum over all permissible sets $\zeta$, we obtain

$$
\|x y\|_{W_{p}}^{p} \leqslant 2^{p-1}\left(\|x\|_{\infty}^{p}\|y\|_{W_{p}}^{p}+\|y\|_{\infty}^{p}\|x\|_{W_{p}}^{p}\right) \leqslant 2^{p}\|x\|_{W_{p}}^{p}\|y\|_{W_{p}}^{p},
$$

as $\|\cdot\|_{W_{p}}$ dominates the supremum norm. So

$$
\|x y\|_{W_{p}} \leqslant 2\|x\|_{W_{p}}\|y\|_{W_{p}} .
$$

Hence for $x, y \in X_{p}$, we have

$$
\begin{aligned}
\|x y\|_{X_{p}}=\sup _{n}\left\|P_{n}(x y)\right\|_{W_{p}} & =\sup _{n}\left\|P_{n}(x) P_{n}(y)\right\|_{W_{p}} \\
& \leqslant 2 \sup _{n}\left(\left\|P_{n} x\right\|_{W_{p}}\left\|P_{n} y\right\|_{W_{p}}\right) \quad \text { by equation }(\dagger) \\
& \leqslant 2\left(\sup _{n}\left\|P_{n} x\right\|_{W_{p}}\right)\left(\sup _{n}\left\|P_{n} y\right\|_{W_{p}}\right)=2\|x\|_{X_{p}}\|y\|_{X_{p}} .
\end{aligned}
$$

This shows that the product $x y$ belongs to $X_{p}$ and is jointly continuous.

The involution $*$ is isometric on $X_{p}$, because $|\alpha-\beta|=|\bar{\alpha}-\bar{\beta}|$ for all $\alpha, \beta \in \mathbb{C}$, so $\nu_{p}\left(P_{n} x, \zeta\right)=\nu_{p}\left(P_{n} x^{*}, \zeta\right)$ for all permissible subsets $\zeta$ of $\mathbb{N}$, and so $\left\|P_{n} x^{*}\right\|_{W_{p}}=$ $\left\|P_{n} x\right\|_{W_{p}}<\infty$ for each $n \in \mathbb{N}$, and hence finally we have $\left\|x^{*}\right\|_{X_{p}}=\|x\|_{X_{p}}<\infty$, therefore $x^{*} \in X_{p}$.

As in Lemma 2.2 , let $\|x\|_{X_{p}}:=\sup \left\{\|x y\|_{X_{p}}: y \in X_{p},\|y\|_{X_{p}} \leqslant 1\right\}$ for $x \in X_{p}$.

Corollary 4.2. The Banach space $X_{p}$ is a commutative unital Banach sequence algebra under the $\|\cdot\|_{X_{p}}$-norm with isometric involution.

Proof. It has been shown in Lemma 4.1 that $X_{p}$ is an algebra with norm $\|\cdot\|_{X_{p}}$ and jointly continuous product, and it is clear that $\chi_{\mathbb{N}}$ is an identity. So by an application of Lemma 2.2 we see that $\|\cdot\|_{X_{p}}$ is a submultiplicative norm on $X_{p}$ equivalent to $\|\cdot\|_{X_{p}}$, and $\left\|\chi_{\mathbb{N}}\right\|_{X_{p}}=1$. The involution $*$ is isometric as $\left\|x^{*}\right\|_{X_{p}}=\|x\|_{X_{p}}$, and so $\left\|x^{*}\right\|_{X_{p}}=\|x\|_{X_{p}}$.

COROLlary 4.3. $W_{p}$ is a closed $*$-ideal in $X_{p}$, and $X_{p}$ is naturally identified with the unitisation of $W_{p}$.

Proof. Let $x \in W_{p}$ and $y \in X_{p}$, then $x y \in c_{0}$ and $\|x y\|_{W_{p}}<\infty$ by equation (†) above, so $x y \in W_{p}$. It is clear that $W_{p}$ is closed under the involution. 
By Lemma 2.3 and by the definition of $V_{p}$ as the closure of $c_{00}$, we have:

Corollary 4.4. $V_{p}$ is a closed $*$-ideal in $X_{p}$.

By Proposition 2.4, we have:

Corollary 4.5. The character space of $V_{p}$ is $\left\{f_{i}: i \in \mathbb{N}\right\}$, that is, $V_{p}$ is natural.

By [2, Remark 4.16] the map $\lambda: X_{p} \rightarrow \mathbb{C}:\left(\alpha_{n}\right)_{n \in \mathbb{N}} \mapsto \lim _{n \rightarrow \infty} \alpha_{n}$, is a linear functional; $\lambda$ is also multiplicative and hence is a character of $X_{p}$, and so $\lambda$ is continuous and $\|\lambda\|=1$. This observation gives an alternative explanation for the boundedness of $\lambda$.

Let $\mathscr{S}_{B}$ denote the state space of a unital Banach $*$-algebra $B$.

Corollary 4.6. The Banach *-algebras $V_{p}, W_{p}$, and $X_{p}$ are $*$-semisimple.

Proof. We have

$$
*-\operatorname{rad} V_{p}=*-\operatorname{rad}\left(V_{p}+\mathbb{C} \chi_{\mathbb{N}}\right)=\left.\bigcap\left\{\operatorname{ker} \lambda: \lambda \in \mathscr{S}_{V_{p}+\mathbb{C} \chi_{\mathbb{N}}}\right\} \subseteq \bigcap_{n \in \mathbb{N}} \operatorname{ker} f_{n}\right|_{V_{p}+\mathbb{C} \chi_{n}}=\{0\}
$$

and

$$
*-\operatorname{rad} W_{p}=*-\operatorname{rad} X_{p}=\left.\bigcap\left\{\operatorname{ker} \lambda: \lambda \in \mathscr{S}_{X_{p}}\right\} \subseteq \bigcap_{n \in \mathbb{N}} \operatorname{ker} f_{n}\right|_{X_{p}}=\{0\} .
$$

Proposition 4.7. The sequence $\left(\chi_{n}\right)$ is a bounded approximate identity of norm-1 projections in the Banach *-algebra $V_{p}$, and is contained in $c_{00}$.

Proof. We may calculate from the definition that $\left\|\chi_{n}\right\|_{W_{p}}=1$ for all $n \in \mathbb{N}$.

We note that by a remark following [5, 4.1.34], Proposition 4.7 implies that the Banach sequence algebra $V_{p}$ is a strong Ditkin algebra, and spectral synthesis holds.

By an application of Proposition 2.5 (i), we have:

Corollary 4.8. The closed ideals of $V_{p}$ are all of the form $I_{V_{p}}(\zeta)$, for some $\zeta \subseteq \mathbb{N}$ and each has an approximate identity.

The statement of the following proposition may be found for the James spaces in [5], 4.1.45 (viii)] or [13, 4.2.3]. With a small alteration to take account of permissibility, these proofs may be adapted for the James-Schreier algebras. However, here we give a slightly different presentation that makes use of a calculation in the proof of [2, 4.18(ii)].

For a strictly increasing mapping $\sigma: \mathbb{N} \rightarrow \mathbb{N}$, we recall from [2, 4.18 and 4.20] the definitions on $\left(c_{00},\|\cdot\|_{V_{p}}\right)$ of: the left shift, $\Lambda_{\sigma}: \sum_{n=1}^{\infty} \alpha_{n} e_{n} \mapsto \sum_{n=1}^{\infty} \alpha_{\sigma(n)} e_{n}$, which is contractive; the right shift, $R_{\sigma}: \sum_{n=1}^{\infty} \alpha_{n} e_{n} \mapsto \sum_{n=1}^{\infty} \alpha_{n} e_{\sigma(n)}$, which is bounded if and only if $\sigma(\mathbb{N})$ is cofinite in $\mathbb{N}$; and the block right shift, $\Theta_{\sigma}: \sum_{n=1}^{\infty} \alpha_{n} e_{n} \mapsto \sum_{n=1}^{\infty} \alpha_{n} \chi_{(\sigma(n-1), \sigma(n)]}$ (with the convention that $\sigma(0)=0$ ), which is bounded if and only if there is a constant $K \in \mathbb{N}$ such that $\sigma(n) \leqslant K n$ for each $n \in \mathbb{N}$. In the bounded case, these shifts extend uniquely to bounded operators on $V_{p}$.

Proposition 4.9. Let $\sigma: \mathbb{N} \rightarrow \mathbb{N}$ be a strictly increasing mapping. Then $I_{V_{p}}(\sigma(\mathbb{N}))$ has a bounded approximate identity if and only if $\sigma(\mathbb{N})$ is cofinite in $\mathbb{N}$.

Proof. We argue as in Proposition 2.5(ii). If $\mathbb{N} \backslash \sigma(\mathbb{N})$ is finite then the approximate identity $\chi_{\sigma((0, n])}=R_{\sigma} \chi_{n}$ is bounded, as the right shift $R_{\sigma}$ is itself bounded. 
Conversely, aiming towards a contradiction, assume that $\mathbb{N} \backslash \sigma(\mathbb{N})$ is infinite and that the ideal $I_{V_{p}}(\sigma(\mathbb{N}))$ has a bounded approximate identity $\left(\omega_{n}\right)$ (necessarily sequential as $V_{p}$ is separable), with $\left\|\omega_{n}\right\|_{V_{p}} \leqslant C$ for some $C \in \mathbb{N}$. We recall that, by a previous calculation in the proof that $R_{\sigma}$ is unbounded [2, 4.18(ii)], we have $\left\|R_{\sigma} \chi_{k}\right\|_{V_{p}} \rightarrow \infty$ as $k \rightarrow \infty$, and hence there exists $k$ such that $\left\|R_{\sigma} \chi_{k}\right\|_{V_{p}} \geqslant C+1$. As $\omega_{n}$ is a bounded approximate identity, there exists $n \in \mathbb{N}$ such that $\left\|R_{\sigma} \chi_{k}-\omega_{n} R_{\sigma} \chi_{k}\right\|_{V_{p}} \leqslant \frac{1}{2}$. Hence

$$
\begin{aligned}
\frac{1}{2} & \geqslant\left\|R_{\sigma} \chi_{k}-\omega_{n} R_{\sigma} \chi_{k}\right\|_{V_{p}} \geqslant\left\|R_{\sigma} \chi_{k}\right\|_{V_{p}}-\left\|\omega_{n} R_{\sigma} \chi_{k}\right\|_{V_{p}}=\left\|R_{\sigma} \chi_{k}\right\|_{V_{p}}-\left\|P_{\sigma(k)} \omega_{n}\right\|_{V_{p}} \\
& \geqslant\left\|R_{\sigma} \chi_{k}\right\|_{V_{p}}-\left\|\omega_{n}\right\|_{V_{p}} \geqslant(C+1)-C=1,
\end{aligned}
$$

which is a clear contradiction.

Proposition 4.10. Let $\sigma: \mathbb{N} \rightarrow \mathbb{N}$ be a strictly increasing mapping such that, for some constant $K \in \mathbb{N}$, we have $\sigma(n) \leqslant K n$ for each $n \in \mathbb{N}$. Then the closed ideal $I_{V_{p}}(\mathbb{N} \backslash \sigma(\mathbb{N})$ ) is complemented in $V_{p}$.

Proof. For such a mapping $\sigma$, the left shift $\Lambda_{\sigma}$ is a contractive operator and the block right shift $\Theta_{\sigma}$ is a bounded operator on $V_{p}$ such that $\Lambda_{\sigma} \Theta_{\sigma}=I_{V_{p}}$. By [2, 3.18], $\Theta_{\sigma} \Lambda_{\sigma}$ and hence $I_{V_{p}}-\Theta_{\sigma} \Lambda_{\sigma}$ are idempotent. Thus $I_{V_{p}}(\mathbb{N} \backslash \sigma(\mathbb{N}))=\operatorname{ker}\left(\Lambda_{\sigma}\right)=\operatorname{im}\left(I_{V_{p}}-\Theta_{\sigma} \Lambda_{\sigma}\right)$ is a complemented subspace of $V_{p}$.

COROLlary 4.11. The Banach algebra $V_{p}$ is weakly amenable but not amenable.

Proof. Proposition 2.7 implies that $V_{p}$ is weakly amenable. By [5, 2.9.59], for an amenable Banach algebra $B$, a closed ideal $I$ is (weakly) complemented in $B$ (if and) only if $I$ has a bounded approximate identity. Hence by Propositions 4.9 and 4.10 , we conclude that $V_{p}$ is not amenable, by noting that, in particular $I_{V_{p}}(2 \mathbb{N})$ is a complemented ideal with no bounded approximate identity.

The proofs above may be paraphrased to say that the James-Schreier algebras are not amenable because there exist strictly increasing mappings $\sigma: \mathbb{N} \rightarrow \mathbb{N}$ such that $\Theta_{\sigma}$ is bounded but $\left(R_{\mathbb{N} \backslash \sigma(\mathbb{N})} \chi_{n}\right)_{n \in \mathbb{N}}$ is not uniformly norm-bounded.

We also note that $V_{p}$ (and $J_{p}$ ) are sequentially approximately contractible. That $V_{p}$ has a bounded sequential approximate identity in $c_{00}$ implies that it is sequentially approximately amenable [6, 3.5], and approximate amenability is equivalent to approximate contractibility by a result of Ghahramani, Loy and Zhang [8].

Proposition 4.12. The Banach *-algebras $W_{p}$ and $V_{p}$ contain complemented closed $*-$ ideals isomorphic to the Banach *-algebras $Z_{p}$ and $S_{p}$ respectively.

Proof. For $x=\left(\alpha_{n}\right)_{n \in \mathbb{N}}$ the mapping $\Phi x:=\left(0, \alpha_{1}, 0, \alpha_{2}, 0, \alpha_{3}, \ldots\right) \in \mathbb{C}^{\mathbb{N}}$ defined in [2, $4.5]$ preserves pointwise multiplication and the involution. Given that the restrictions of $\Phi$ define isomorphisms from $Z_{p}$ to a subspace of $W_{p}$, and from $S_{p}$ to a subspace of $V_{p}$ respectively, then these are evidently $*$-isomorphisms. The image of $Z_{p}$ under $\Phi$ is the complemented ideal $\Phi\left(Z_{p}\right)=I_{W_{p}}(2 \mathbb{N})$, and similarly, $\Phi\left(S_{p}\right)=I_{V_{p}}(2 \mathbb{N})$.

This gives an alternative proof that $V_{p}$ is not amenable, as $S_{p} \cong I_{V_{p}}(2 \mathbb{N})$ is a complemented ideal of $V_{p}$, and $S_{p}$ is not amenable.

By Lemma 2.8, as $V_{p}$ is a Banach *-algebra with a shrinking basis, we have: 
Corollary 4.13. The two Arens products on $V_{p}^{\prime \prime}$ (identified with $X_{p}$ via $\Gamma$ ) coincide with the product in $X_{p}$. Hence $V_{p}$ is Arens regular, and $V_{p}^{\prime \prime}$ with the Arens product is *-isomorphic to $X_{p}$.

The multiplier algebra of a commutative faithful Banach algebra $B$, is given by $M(B):=\{T: B \rightarrow B: a T(b)=T(a) b \quad(a, b \in B)\}$. When $B$ has an isometric involution $*$, we equip $M(B)$ with an involution $T \mapsto T^{\triangleleft}$ given by $T^{\triangleleft} x:=\left(T\left(x^{*}\right)\right)^{*}$ for all $x \in B$, as in [5, 1.10.1]. A Banach sequence algebra contains $c_{00}$ and hence is always faithful.

Proposition 4.14. The multiplier algebra $M\left(V_{p}\right)$ is $*$-isomorphic to $X_{p}$.

Proof. For $x \in X_{p}$ and $a \in V_{p}$, let $L_{x}(a):=x a$; we have $x a \in V_{p}$ as $V_{p}$ is an ideal of $X_{p}$, and so $L_{x} \in M\left(V_{p}\right)$ by commutativity of $V_{p}$. We claim that $\mathscr{L}: X_{p} \rightarrow M\left(V_{p}\right): x \mapsto L_{x}$ is a $*$-isomorphism.

It follows straightforwardly from the definition that $L_{x}^{\triangleleft}(a)=x^{*} a=L_{x^{*}}(a)$ for all $a \in V_{p}$. Continuity of $\mathscr{L}$ is automatic by the Banach algebra inequality: for $x \in X_{p}$ we have $\|\mathscr{L} x\| \leqslant\|x\|_{X_{p}}$. To see that $\mathscr{L}$ is injective, suppose $L_{x}=0$. Then $L_{x} a=x a=0$ for all $a \in V_{p}$. In particular, $x e_{n}=0$ for all $n \in \mathbb{N}$, which implies that $x=0$.

We finally show surjectivity: suppose $T \in M\left(V_{p}\right)$. We have $T\left(e_{j}\right) e_{k}=T\left(e_{j}\right) e_{k} e_{k}=$ $e_{j} T\left(e_{k}\right) e_{k}=0$ for all $j \neq k$. Hence for all $k \in \mathbb{N}$, we deduce that $T\left(e_{k}\right)=\alpha_{k} e_{k}$ for some $\alpha_{k} \in \mathbb{C}$. Let $x:=\left(\alpha_{n}\right)_{n \in \mathbb{N}}$. We claim that $x \in X_{p}$ and that $T=L_{x}$. By linearity of $T$, we have $\chi_{n} x=T\left(\chi_{n}\right)$, and so

$$
\left\|P_{n} x\right\|_{V_{p}}=\left\|\chi_{n} x\right\|_{V_{p}}=\left\|T\left(\chi_{n}\right)\right\|_{V_{p}} \leqslant\|T\|\left\|\chi_{n}\right\|_{V_{p}}=\|T\|,
$$

hence $\|x\|_{X_{p}}=\sup _{n}\left\|P_{n} x\right\|_{V_{p}}<\infty$ so $x \in X_{p}$. It is sufficient to check $T=L_{x}$ by evaluation on the basis $\left(e_{n}\right)_{n \in \mathbb{N}}$ of $V_{p}$ : we have $T\left(e_{i}\right)=\alpha_{i} e_{i}=L_{x}\left(e_{i}\right)$.

COROLlary 4.15. The multiplier algebra $M\left(W_{p}\right)$ is $*$-isomorphic to $X_{p}$.

Proof. The proof of Proposition 4.14 may be repeated with $W_{p}$ in the place of $V_{p}$ to show that $M\left(W_{p}\right)$ is $*$-isomorphic to $X_{p}$-though whereas before in the proof of surjectivity it sufficed to check that $T$ and $L_{x}$ agree on each basis vector $e_{i}$ of $V_{p}$, now as $W_{p}$ has no basis we must show that $T(a)=L_{x}(a)$ for all $a \in W_{p}$. We have $e_{i} T(a)=T\left(e_{i}\right) a=$ $\alpha_{i} e_{i} a=x e_{i} a=e_{i} L_{x}(a)$ for all $i \in \mathbb{N}$, hence $T(a)$ and $L_{x}(a)$ agree on every coordinate, so they are equal.

As $X_{p}$ is a commutative Banach algebra with identity, $M\left(X_{p}\right) \cong X_{p}[9$. Furthermore, as each multiplier $T \in M\left(X_{p}\right)$ is given by $T=L_{T\left(\chi_{\mathbb{N}}\right)}$, the isomorphism is clearly a *-isomorphism as in the proof of Proposition 4.14

COROLlary 4.16. The following five Banach $*$-algebras: $X_{p}$; the bidual $V_{p}^{\prime \prime}$ with the Arens product; and the multiplier algebras $M\left(V_{p}\right), M\left(W_{p}\right)$, and $M\left(X_{p}\right)$; are *-isomorphic.

Extending a result in [10, White classified the closed ideals of the James algebra $J_{p}$ in [13]. However, in [7] it was shown that there is an uncountable family $\mathscr{F}$ of subsets of $\mathbb{N}$ such that the Banach spaces $I_{S_{1}}(\zeta)$ and $I_{S_{1}}(\xi)$ do not embed in each other whenever $\zeta, \xi \in \mathscr{F}$ are distinct. This suggests that a corresponding classification of the closed ideals for $V_{1}$ would be difficult. 
Acknowledgements. This paper is based on material delivered in a lecture at the $19^{\text {th }}$ International Conference on Banach Algebras held at Będlewo, July 14-24, 2009. The author would like to thank all the conference organisers for their superb work, and those who provided support: the Polish Academy of Sciences; the European Science Foundation under the ESF-EMS-ERCOM partnership; and the Faculty of Mathematics and Computer Science of the Adam Mickiewicz University at Poznań. The author is also grateful for the funding of his doctoral studies by the EPSRC (EP/P500303/1, EP/P501482/1, and $\mathrm{EP} / \mathrm{P} 502656 \mathrm{X} / 1$ ), and to N. J. Laustsen for his help in the preparation of this paper.

\section{References}

[1] A. D. Andrew and W. L. Green, On James' quasi-reflexive space as a Banach algebra, Can. J. Math. 32 (1980), 1080-1101.

[2] A. Bird and N. J. Laustsen, An amalgamation of the Banach spaces associated with James and Schreier, Part I: Banach-space structure, this volume, 45-76.

[3] A. Bird, N. J. Laustsen, and A. Zsák, Some remarks on James-Schreier spaces, J. Math. Anal. Appl. 371 (2010), 509-613.

[4] F. F. Bonsall and J. Duncan, Complete Normed Algebras, Springer-Verlag, 1973.

[5] H. G. Dales, Banach Algebras and Automatic Continuity, Oxford Sci. Pub., 2000.

[6] H. G. Dales, R. J. Loy, and Y. Zhang, Approximate amenability for Banach sequence algebras, Studia Math. 177 (2006), 81-96.

[7] I. Gasparis and D. H. Leung, On the complemented subspaces of the Schreier spaces, Studia Math. 141 (2000), 273-300.

[8] F. Ghahramani, R. J. Loy and Y. Zhang, Generalized notions of amenability II, J. Funct. Anal. 254 (2008), 1776-1810.

[9] R. Larsen, An Introduction to the Theory of Multipliers, Springer-Verlag, 1971.

[10] N. J. Laustsen, Maximal ideals in the algebra of operators on certain Banach spaces, Proc. Edin. Math. Soc. 45 (2002), 523-546.

[11] R. E. Megginson, An Introduction to Banach Space Theory, Springer-Verlag, 1998.

[12] T. W. Palmer, Banach Algebras and the General Theory of *-Algebras, Volume I, Algebras and Banach Algebras, CUP, 1994.

[13] K. A. L. White, Derivations and intertwining maps from Banach algebras, Ph.D. Thesis, Univ. of Leeds, 2000.

[14] K. A. L. White, Amenability and ideal structure of some Banach sequence algebras, J. London Math. Soc. 68 (2003), 444-460. 
